\title{
Transcriptome and expression profiling analysis link patterns of gene expression to antennal responses in Spodoptera litura
}

\author{
Bo Feng ${ }^{1+}$, Xinda Lin ${ }^{2 \dagger}$, Kaidi Zheng ${ }^{1}$, Kai Qian', Yongchang Chang ${ }^{3}$ and Yongjun Du ${ }^{1 *}$
}

\begin{abstract}
Background: The study of olfaction is key to understanding the interaction of insects with their environment and provides opportunities to develop novel tactics for control of pest species. Recent developments in transcriptomic approaches enable the molecular basis of olfaction to be studied even in species with limited genomic information. Here we use transcriptome and expression profiling analysis to characterize the antennal transcriptome of the noctuid moth and polyphagous pest Spodoptera litura.

Results: We identify 74 candidate genes involved in odor detection and recognition, encoding 26 ORs, 21 OBPs, 18 CSPs and 9 IRs. We examine their expression levels in both sexes and seek evidence for their function by relating their expression with levels of EAG response in male and female antennae to 58 host and non-host plant volatiles and sex pheromone components. The majority of olfactory genes showed sex-biased expression, usually male-biased in ORs. A link between OR gene expression and antennal responses to odors was evident, a third of the compounds tested evoking a sex-biased response, in every case also male-biased. Two candidate pheromone receptors, OR14 and OR23 were especially strongly expressed and male-biased and we suggest that these may respond to the two female sex pheromone components of S. litura, Z9E11-14:OAC and Z9E12-14:OAC, which evoked strongly male-biased EAG responses.
\end{abstract}

Conclusions: Our results provide the molecular basis for elucidating the olfactory profile of moths and the sexual divergence of their behavior and could enable the targeting of particular genes, and behaviors for pest management.

Keywords: Olfactory receptor, RT-qPCR, Electroantennogram, Sex-biased expression, Sex pheromone, Plant volatiles

\section{Background}

Olfaction plays a key role in the interactions of insects with their environment, mediating foraging, aggregation, mating, and oviposition behaviors. Studies of insect olfaction have provided fundamental insights into chemosensory biology and chemical ecology [1-4] and have presented valuable opportunities for pest management [5-8]. Lepidoptera are a focus of interest for studies of olfaction as they have large and sensitive olfactory repertoires [8] yet molecular studies of olfaction in Lepidoptera lag behind those in standard insect models. Recently, there has been exciting progress in identifying

\footnotetext{
*Correspondence: dyj@wzmc.edu.cn

${ }^{\dagger}$ Equal contributors

'Institute of Health and Environmental Ecology, Wenzhou Medical University, University Town, Wenzhou 325035, China

Full list of author information is available at the end of the article
}

genes coding for lepidopteran olfactory receptors, not only in the model Bombyx mori [8-10], for which there is genomic data [11], but also in the pest species Manduca sexta [12], Heliothis virescens [13,14] and Spodoptera frugiperda [15], however it's a draft assembly to present, which would be a better reference for S.litura in the future. Progress in the absence of genomic data has been made possible by genome-wide approaches for transcriptome analysis, such as RNA-Sequencing (RNASeq) [12]. Such high-throughput molecular techniques and associated informatics technologies, are becoming commonplace in chemical ecology $[9,16,17]$.

A sufficient level of expression of genes is key to the success of transcriptomic approaches to their identification. In an elegant and comprehensive study on the antennal transcriptome of $M$. sexta, Grosse-Wilde et al. [12] identified the main olfactory genes and compared 
their expression in males and females. In an equally thorough study of the antennal transcriptome of S. littoralis, Jacquin-Joly et al. $[18,19]$ examined the expression of 7 olfactory and 4 gustatory receptors in different tissues and discussed their function. They suggested that transcriptome expression may change following mating and could reveal more olfactory genes involved in sexspecific behavior. If expression levels of olfactory genes could be linked with functional responses to volatiles, expression profiling could lead to a better understanding of the function and operation of olfactory genes and could elucidate how individual variation of olfactory gene expression might lead to speciation or resistance to pheromonal pest management.

Olfactory neurons express many proteins involved in the capture of volatiles from the environment and signal transduction. These include olfactory receptors (ORs) [14,19-21], odorant-binding proteins (OBPs) [22,23], chemosensory proteins (CSPs) [23,24] and ionotropic receptors (IRs) [25]. ORs specifically bind odorant molecules and initiate signal transduction in the membrane of the olfactory neuron. Insect ORs generally exhibit low levels of homology and are selectively expressed in olfactory neurons at low levels [26]. Members of the OR83b receptor subfamily, commonly known as olfactory receptor coreceptors (ORCOs), are more conserved and expressed in most olfactory neurons at various stages of development [27]. In Lepidoptera, OBPs are classified into pheromone-binding proteins (PBPs) [28], general odorant-binding proteins (GOBPs) $[29,30]$ and antennalbinding proteins (ABPs) [31]. ABPs are expressed specifically in the antenna with characteristics typical of OBPs $[29,32,33]$ but they have low homology with PBPs and GOBPs and their function remains unknown. The CSPs constitute a conserved family of binding proteins that are unrelated to OBPs and whose function is again unclear $[34,35]$. IRs, recently described novel family of olfactory receptors [36], are localized on the dendrite of chemosensory neurons and are ligand-gated ion channels that mediate chemical communication between neurons [37]. IRs were further classified into two subfamilies: conserved "antennal IRs" involved in olfaction and species-specific "divergent IRs" that might be associated with gustation $[37,38]$.

The tobacco cutworm moth, Spodoptera litura, is an important agriculture pest widely distributed throughout tropical and temperate Asia, Australia and the Pacific Islands (Additional file 1: Figure S1), noctuid moth and a polyphagous pest with more than 290 host plants belonging to 99 families [39]. The S. litura attacks numerous economically important crops and trees, it also defoliated these crops or trees, finally leads to serious economic yield loss [40]. Its two-component sex pheromone has been identified [41], together with a plant- derived synergist [42], and the pheromone is used at a large scale for mass trapping for pest management [42]. However, little is known about S. litura's ORs, CSPs and OBPs [43-46]. Previous behavioral bioassays have demonstrated that male and female moths respond differently to odorants, including pheromones [41]. The complete genome of $S$. litura is not yet available.

Here we study the molecular mechanisms underlying sex-specific response to odors, including sex pheromones, in S. litura, and discuss the link between OR gene expression and chemosensory responses as measured by electroantennography. Using de novo transcriptome and expression profile analysis we achieved a high level of coverage of olfactory genes and measured gene expression using both single-end RNA-Seq and RT-qPCR to give confidence in comparisons between sexes at lower expression levels. We discuss the implications of our study for understanding the functioning of olfactory genes.

\section{Results}

\section{Olfactory responses of male and female moths}

The antennae of both sexes showed varying electroantennogram (EAG) responses to the 58 chemicals that were presented, representing flower volatiles, host or non-host plant volatiles, and S. litura sex pheromone components and their isomers (Figure 1 and Additional file 1: Figure S2). For 11 floral odors, two plant volatiles, and six sex pheromone components or isomers EAG responses differed significantly between sexes, and in each case males responded more strongly (Figure 1). In male antennae there was a positively dose-dependent response to $S$. litura sex pheromone gland components, both by those that elicit behavioural responses, $(9 \mathrm{Z}, 11 \mathrm{E})$-tetradecadienyl acetate (Z9E11-14:OAc) and (9Z,12E)-tetradecadienyl acetate (Z9E12-14:OAc), and also by the minor components 9Z-tetradecenyl acetate (Z9-14:OAc) and 9E-tetradecenyl acetate (E9-14:OAc) (Figure 1A and B). The sex pheromone isomers 11E-tetradecenyl acetate (E11-14:OAc) and 11Z-tetradecenyl acetate (Z11-14: OAc) elicited significant EAG responses that differed between sexes at the $10^{-2}$ dosage $(\mathrm{v} / \mathrm{v})$ although they are not found in female moths.

\section{De novo transcriptome assembly}

A total of 55,288,304 reads of the pooled RNA extract were generated through Illumina sequencing and assembled into 105,971 contigs and then 69,301 unigenes, with a mean length of $603 \mathrm{bp}$ (Additional file 1: Figure S3). More than $39 \%$ of all unigenes aligned to sequences in protein databases. The gene ontology (GO) annotation provides information of the gene products, the molecular function, biological process involved and the cellular location. GO annotation makes the transcriptome data more 


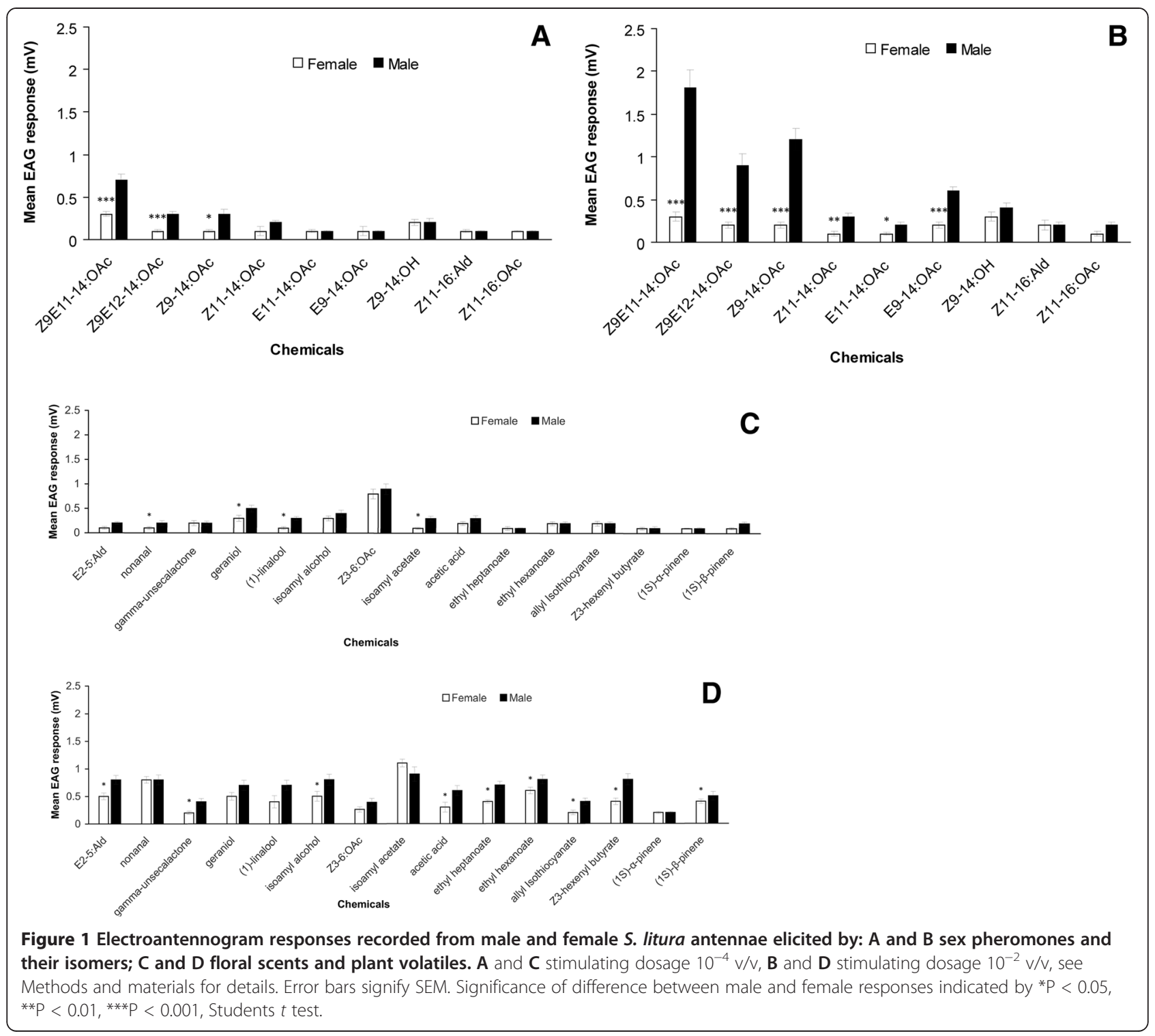

accessible and was used to assess the transcriptome (Additional file 1: Figure S4).

\section{Analysis of olfactory genes}

Twenty six putative OR genes, 21 OBPs, 18 CSPs and 9 IRs were identified for $S$. litura and the mean length of OR, OBP, CSP and IR was 335 aa, 147 aa, 132 aa and 644 aa separately. Phylogenetic comparison revealed that 24 (except for OR44 and OR45) of S. litura ORs clustered with verified ORs of Lepidoptera (bootstrap value $\geq 50$ ) (Additional file 1: Figure S5). Spodoptera litura ORCO clustered with the ORCO subfamily, SlituOR18 clustered with other lepidopteran OR18 and 5 ORs (OR1, OR11, OR13, OR14, OR23) fell into the pheromone receptors subfamily. Except for the ORCO, ORs of different orders
(Lepidoptera, Diptera, Hymenoptera and Hemiptera) were diverged. The twenty-one OBP genes encode 11 OBPs, 4 ABPs, 2 GOBPs, 3 PBPs, and one ABPX. Except for SlituOBP6, all S. litura OBP genes were clustered with those of Lepidoptera (bootstrap value $\geq 50$ ) (Additional file 1: Figure S6). OBPs of different order (Lepidoptera, Diptera, Hymenoptera and Hemiptera) were also diverged. Of the 18 CSP genes of S. litura (CSP1-18), 16 (except for CSP2 and CSP3) clustered exclusively (bootstrap value $\geq 70$ ) with CSPs of Lepidoptera (Additional file 1: Figure S7). One conserved S. litura CSP (SlituCSP1) occupied clade with CSPs of A. mellifera, A. pisum and other Lepidoptera. Nine IRs of S. litura were clustered with those of Lepidoptera (bootstrap value $\geq 70$ ) (Additional file 1: Figure S8). Meanwhile, IR8a and iGLUR6 
and their lepidopteran analogues were clusterd with those of $D$. melanogaster with high bootstrap values $(\geq 90)$.

\section{Assessment of gene expression in antennae by single-end RNA-Seq}

About 6 million clean reads from the single-end RNASeq library of each of male and female antennal RNA extracts were generated through Illumina sequencing and, of these, $69.3 \%$ and $73.3 \%$, respectively, were uniquely matched with the de novo library (Additional file 1: Table S2). For many olfactory genes where RNA-seq reported low gene expression levels, as measured by RPKM values, and where P-values and false discovery rates (FDR) were $>0.05$ and/or 0.01 separately, estimates of sex differences in relative expression from RT-qPCR and RNA-Seq differed by a factor of two or more. In these circumstances RT-qPCR gives a more reliable measure and was used in preference. The RT-qPCR is generally considered an efficient, fast, reproducible, reliable and specific for quantifying levels of transcripts
[47]. Two reference genes (GAPDH and UCCR) [48] were chose to perform RT-qPCR according to the MIQE guideline [49] and were used to normalize the data in our study.

\section{Expression of all olfactory genes in male and female antennae}

Expression levels of putative OR genes were low. The RPKM values for all ORs were less than 70, except for ORCO which had values of 179 and 262 for female and male antennae, respectively (Figure 2A). Four of the sex differences of ORs in expression shown by RNA-Seq were confirmed by RT-qPCR (Figure 2). Most of sex differences of ORs in expression shown by RNA-Seq were confirmed by RT-qPCR (Figure 2). Of 21 recognized general ORs, RT-qPCR showed 2 to be significantly more expressed in female antennae, relative to the standard GAPDH and $U C C R$ gene, and 170 were significantly more expressed in male antennae (Figure 2B). Like other ORs, expression levels of candidate pheromone receptors measured in the RNA-Seq analysis were

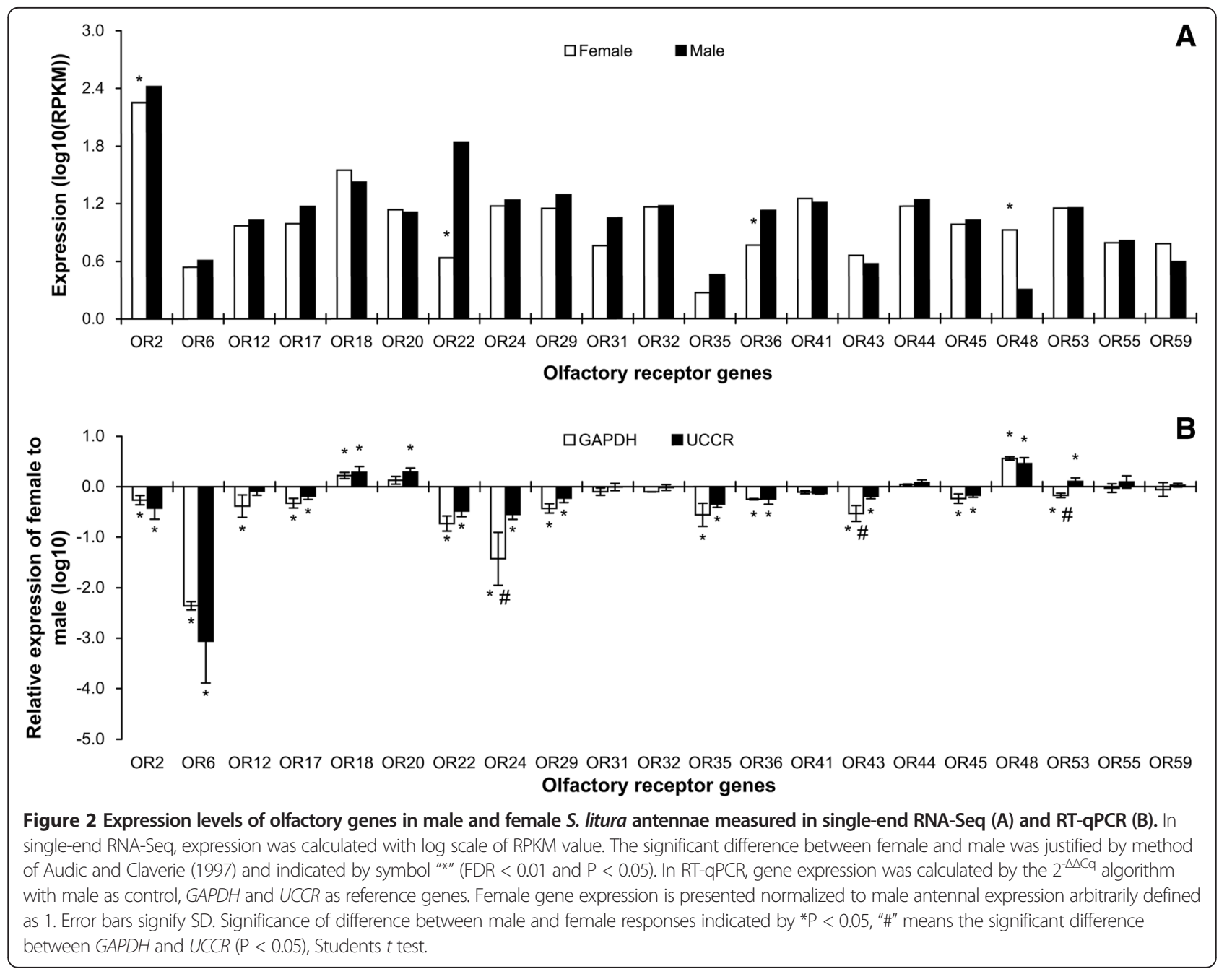


low ( $\leq 80$ RPKM) (Figure 3A). However, expression of OR23 and OR14 in males was markedly higher than for the other 3 candidate pheromone receptors (Figure 3A) and expression of OR14 was amongst the most malebiased. RT-qPCR confirmed the markedly higher relative expression of all candidate pheromone receptors in male antennae (Figure 3B). Usually the difference of general ORs between the sexes was less than four fold. However, OR6 was predominantly expressed in the male compared to the female antennae (Figure 4).

By contrast with candidate pheromone receptors, the expression levels of candidate pheromone-binding protein (PBP) genes in antennae were very high, the male expression of PBP1 being the highest expression of all olfactory genes at 94161 RPKM (Figure 3A). The sexbias expression varied greatly between the three PBPs, being strongly male-biased for PBP1 and female biased for PBP3 (Figure 3A). The levels of expression of other odorant binding proteins in the antennae were extremely variable, with RPKM values ranging from less than 50 to over 20,000 (Figure 5A). Five OBPs were shown to be more highly expressed in female antennae and 3 OBPs to be more highly expressed in male antennae in single- end RNA-Seq and RT-qPCR (Figure 5). However, the total number of male-biased OBPs were the same as that of female (Figure 4). The range of expression levels of CSP genes in the antennae was as extreme as for OBPs, RPKM values ranging from less than 10 to almost 20,000 (Figure 6A). Twelve CSP genes showed sex differences in their levels of antennal expression, 5 being more expressed in females and 7 more expressed in males and sometimes these differences were marked (Figure 6). The expression levels of IR genes were as low as those of ORs and the largest RPKM value was about 1000 (Figure 7A). Of which, IGluR1 was female biased and IR8a was male biased (Figure 7). Only the expression levels of 3 ORs, $P B P 2$ and CSP5 showed significant difference by using GAPDH and $U C C R$ as reference genes.

\section{Discussion}

Spodotera litura is a polyphagous and widely distributed agricultural pest that shows antennal responses to a broad range of chemicals. Until now the genes encoding its olfaction-related proteins have been little known. In this paper we have characterized the olfactory genes of
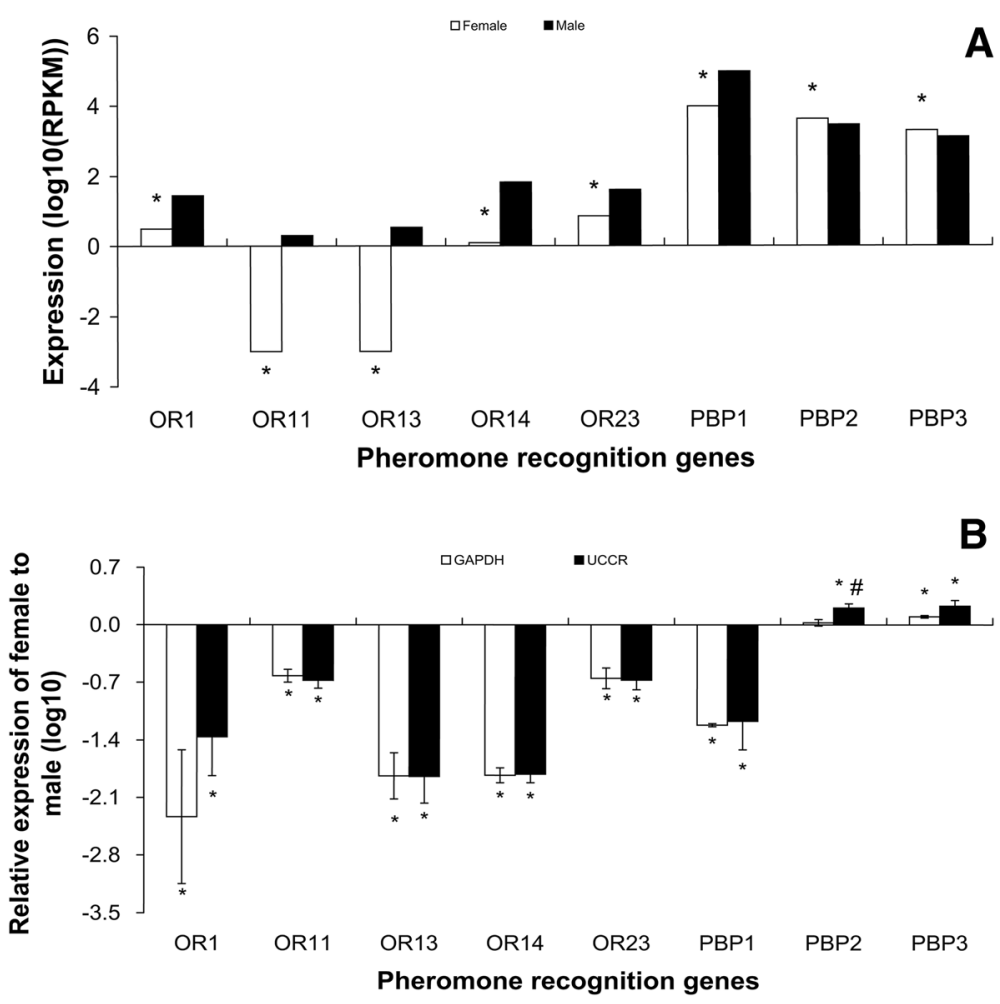

Figure 3 Expression levels of pheromone recognition genes in male and female S. litura antennae measured in single-end RNA-Seq (A) and RT-qPCR (B). In single-end RNA-Seq, expression was calculated with log scale of RPKM value. The significant difference between female and male was justified by method of Audic and Claverie (1997) and indicated by symbol "**" (FDR $<0.01$ and P $<0.05$ ). In RT-qPCR, gene expression was calculated by the $2^{-\triangle C C}$ algorithm with male as control, GAPDH and UCCR as reference genes. Female gene expression is presented normalized to male antennal expression arbitrarily defined as 1. Error bars signify SD, Significance of difference between male and female responses indicated by *P $<0.05$, "\#" means the significant difference between GAPDH and UCCR $(P<0.05)$, Students $t$ test. 


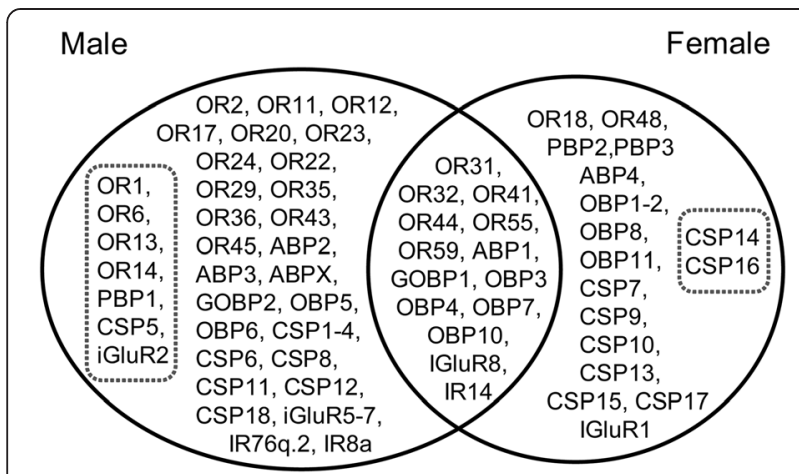

Figure 4 Summary of differences between male and female $S$. litura in the levels of antennal expression of candidate olfactory genes based on RT-qPCR. Genes in the overlapping intersect between sexes show no significant difference between male and female. Genes outside the intersect between sexes show significant difference between sexes. Those in the dash-outlined area show more than tenfold greater expression in male or female antenna and can be considered sex-specific. OR53 and OBP9 were not included because of contradictory results of two reference genes or RT-qPCR failed.
S. litura and their antennal expression using transcriptome analysis, single-end RNA-Seq, and RT-qPCR. We found 74 olfactory genes in the antennae, including 26 ORs, 21 OBPs, 18 CSPs and 9 IRs. Antenal transcriptome was reported in the moths $M$. sexta (48 ORs, 18 OBPs, 21 CSPs and 4 IRs) [12] and B. mori (71 ORs, 20 OBPs, 16 CSPs and 31 IRs) $[11,25]$. In the congeneric species, S. littoralis, 46 ORs, 35 OBPs, 21 CSPs and 5 IRs have recently been found in antennae transcriptome $[18,50,51]$. The surprisingly large difference in the number of olfactory genes obtained for the two Spodoptera species may not be due to the database size but the methodological differences of olfactory genes analysis in the two studies. The S. littoralis study obtained 77,643 contigs with a median size of $653 \mathrm{bp}[18,51]$. In our study, it was 105,971 contigs with a median size of 645 bp, which assembled into 69,301 unigenes with a median size of $974 \mathrm{bp}$. We adopted a more strict criteria to select candidate olfactory genes, a 50\% average ORF length (1200 bp) cutoff as a putative gene. The mean length of OR, OBP, CSP and IR genes was 335 aa, 147 aa, 132 aa and 644 aa respectivelyin our study, which was close to the full length of each category.
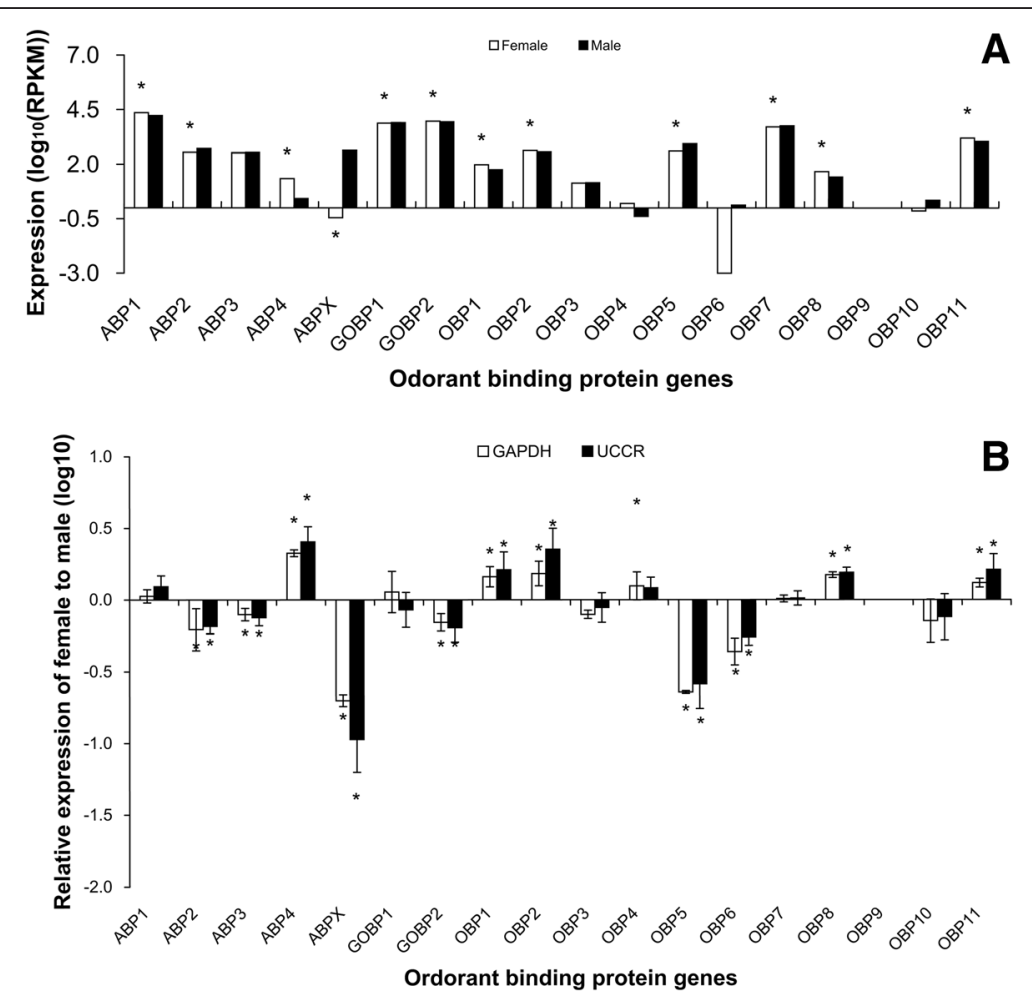

Figure 5 Expression levels of candidate OBPs in male and female S. litura antennae measured in single-end RNA-Seq (A) and RT-qPCR (B). In single-end RNA-Seq, expression was calculated with log scale of RPKM value. The significant difference between female and male was justified by method of Audic and Claverie (1997) and indicated by symbol "*" (FDR $<0.01$ and $P<0.05)$. In RT-qPCR, gene expression was calculated by the $2^{-\triangle \Delta C q}$ algorithm with male as control, GAPDH and UCCR as reference genes. Female gene expression is presented normalized to male antennal expression arbitrarily defined as 1. Error bars signify SD, significance of difference between male and female responses indicated by *P $<0.05$. 

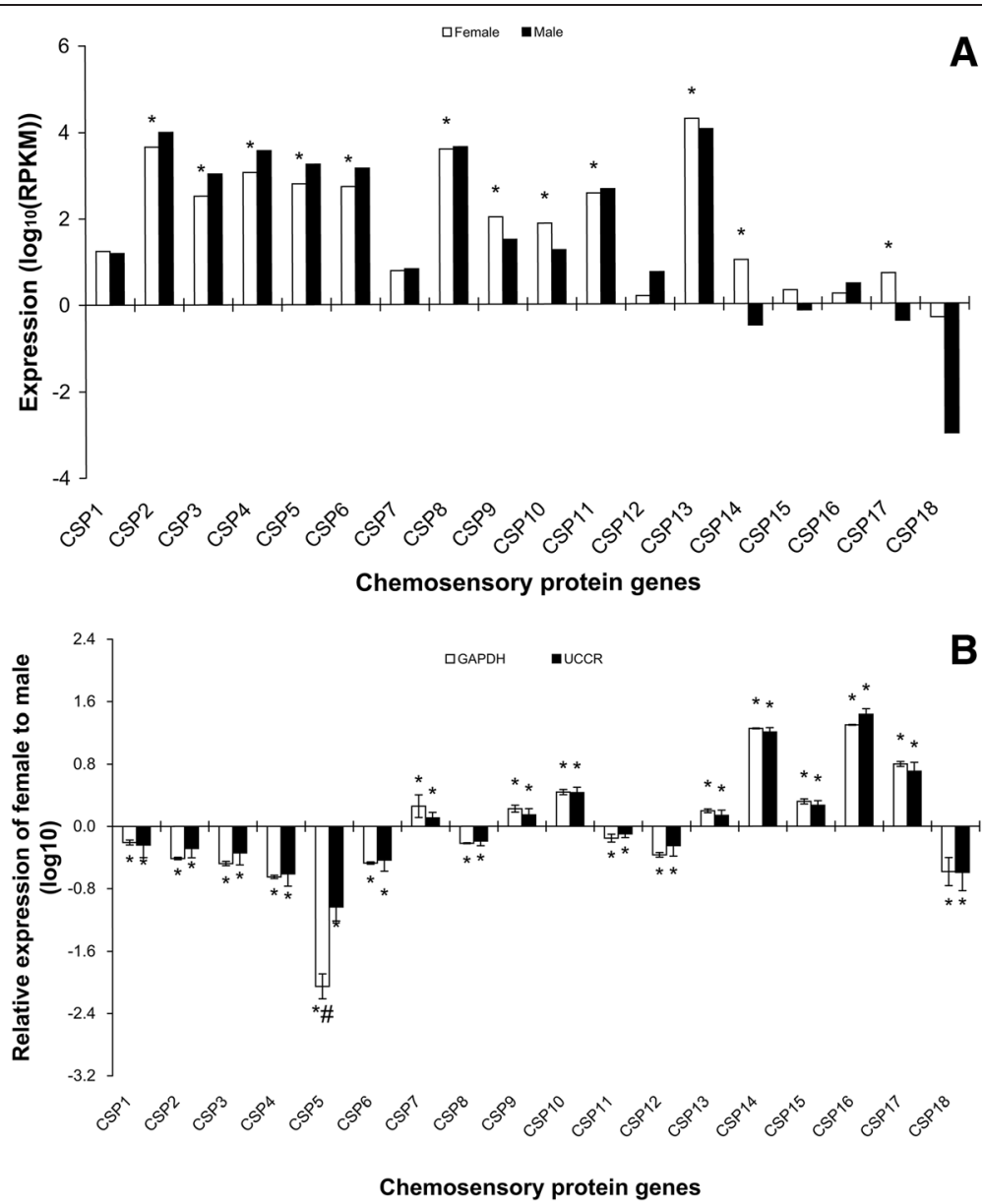

Figure 6 Expression levels of candidate CSPs in male and female S. litura antennae measured in single-end RNA-Seq (A) and RT-qPCR (B). In single-end RNA-seq, expression was calculated with log scale of RPKM value. The significant difference between female and male was justified by method of Audic and Claverie (1997) and indicated by symbol "*" (FDR < 0.01 and P < 0.05). In RT-qPCR, gene expression was calculated by the $2^{-\triangle \triangle C a}$ algorithm with male as control, GAPDH and UCCR as reference genes. Female gene expression is presented normalized to male antennal expression arbitrarily defined as 1. Error bars signify SD. Significance of difference between male and female responses indicated by * $P<0.05$, "\#" means the significant difference between GAPDH and UCCR $(P<0.05)$, Students $t$ test.

Phylogenetic analysis showed that some $S$. litura olfactory genes clustered not only with those of other Lepidoptera but also with those of A. mellifera, D. melanogaster and A. pisum indicating a certain degree of conservation typical of olfactory gene families. The extent of gene conservation is likely to reflect function. For example, the role of OBPs is to assist hydrophobic odorant molecules to cross the aqueous barrier at the pore of the sensillum to reach and bind to ORs on the dendrite of the olfactory neuron [13]. OBPs are relatively generalist, binding more than one molecule [52], and so they are likely to be conserved across larger taxonomic groupings. A recent study by McKenzie et al. showed that both OBPs and CSPs expressed specifically in antennae of the Cerapachys biroi possibly serve the olfactory functions [53]. By contrast, ORs often specifically respond to one particular odorant and those that play a key roles in the autecology of a species are likely to be less conserved across taxa, particularly if they contribute to the reproductive isolation of species as do sex pheromone receptors [54]. ORs that respond to odors common across habitats such as certain green leaf volatiles may be more conserved.

The phylogenetic distribution of S. litura ORs was consistent with other Lepidoptera and the relatively conserved nature of much of the OR gene family. Five $S$. litura ORs clustered together with a conserved OR subfamily of moth sex pheromone receptors [55] on an exclusively lepidopteran branch of the phylogenetic tree. S. litura ORCO clustered with the conserved ORCOs, co-receptors for odorand pheromone-specific ORs [56]. The noctuid moths have another conserved subfamily, OR18 [54]. The OBP family comprises OBPs, ABPs and three subfamilies conserved within Lepidoptera PBP, GOBP, and ABPX $[30,57]$. S. litura OBPs fell both within and outside these subfamilies and clustered with other Lepidoptera. One of S. litura's 18 CSPs clustered with a CSP subfamily which is highly conserved 

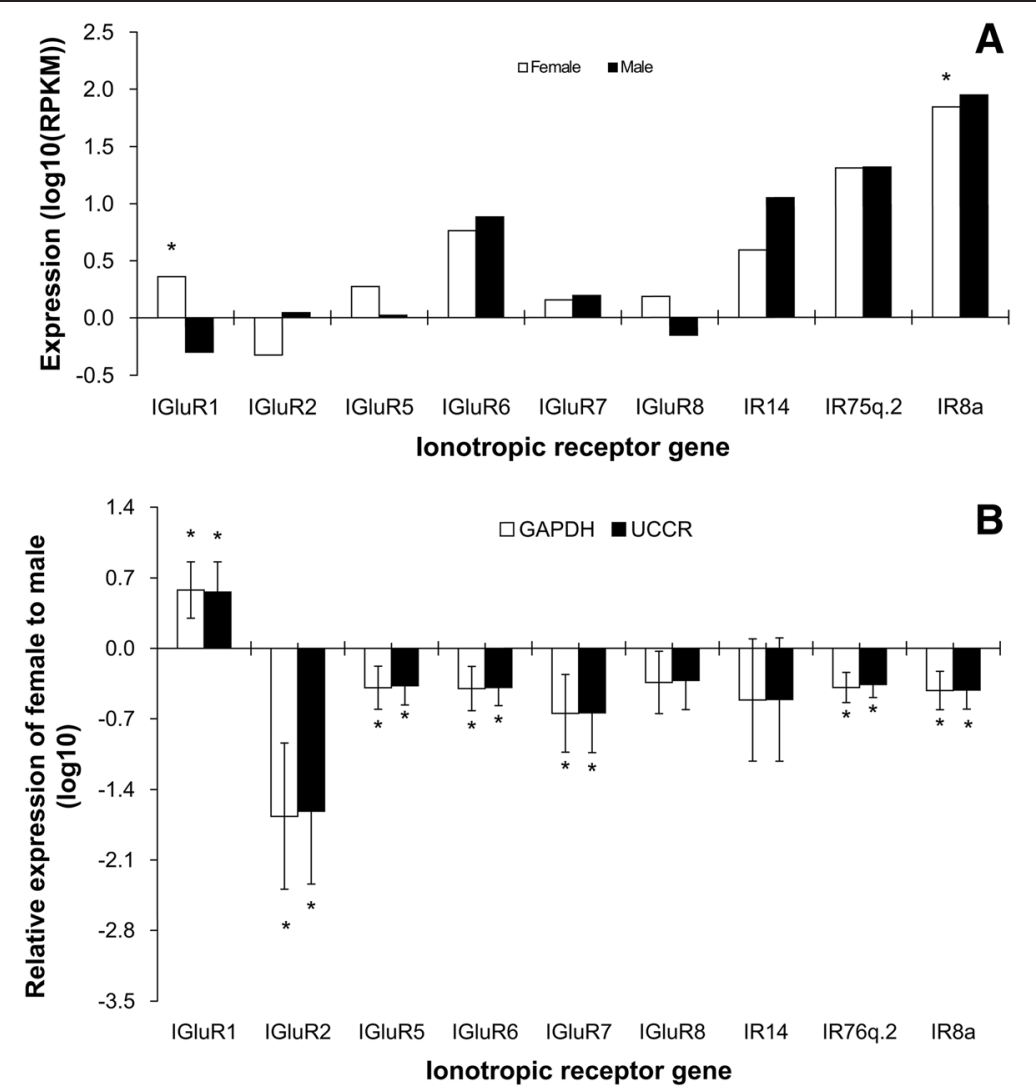

Figure 7 Expression levels of candidate IRs in male and female S. litura antennae measured in single-end RNA-seqence (A) and RTqPCR (B). In single-end RNA-seqence, vertical axis means the log scale of RPKM value to 10. The significant difference between female and male was justified by method of Audic and Claverie (1997) and indicated by symbol "**" (FDR $<0.01$ and P $<0.05$ ). In RT-qPCR, vertical axis means log scale of female gene expression to 10 . Female gene expression was calculated by the $2^{-\triangle \triangle C a}$ algorithm with male as Control and GAPDH and UCCR as reference genes. The significant difference was ascertained by Students $t$ test. Symbol "** means the significant difference between female and male $(P<0.05)$. Error bars signify SD".

across insects and the remainder clustered with lepidopteran CSPs.

In $S$. litura we found the number of candidate pheromone receptor genes (five) close to there are components of the sex pheromone gland (Z9E11-14:OAc, Z9E12-14: OAc, Z9-14:OAc, and E9-14:OAc) that are active in EAG [41]. Multiple sex pheromone receptors (e.g. [35,52,58] and multi-component sex pheromones (e.g. [59-61]) are found in other moths and the excess of pheromone receptor genes is not unique to $S$. litura [59]. Sex pheromone receptors are very specific and so one might expect their number to equal the number of sex pheromone components. It is possible that multiple pheromone receptors may be involved in identifying each component. Using heterologous expression in Xenopus oocytes, both SexiOR13 and SexiOR16 of S. exigua respond to Z9E12-14:OH [62]. Alternatively, during evolution some components may have been lost from the pheromone gland before the corresponding receptor was lost from the antenna [63]. This might explain the EAG response in S. litura to Z11-14:OAc and E11-14: OAc, compounds related to known components of the sex pheromone gland but not themselves present. Retention of the ability to recognise pheromone components that no longer signify conspecific females may assist in the maintenance of reproductive isolation of species.

There were dramatic differences in levels of expression of the diverse olfactory genes in S. litura and some of these can readily be related to function. The 15 most highly expressed genes (RPKM larger than 1000) were all binding proteins (OBPs, CSPs and PBPs). By contrast, most ORs and IRs had RPKM values less than 50. OBPs are usually highly expressed and solubilizing in sensillar lymph. OBPs bind with multiple odorants, and are fewer in number. The most highly-expressed binding protein was a pheromone-binding protein, $P B P 1$, and candidate pheromone receptors were among the most highlyexpressed ORs. This reflects the value of being able to detect very small amounts of pheromone [59]. The most highly expressed OR was ORCO, consistent with evidence from phylogenetic studies [45].

We found many sex differences in expression levels of olfactory genes in S. litura, some of them extreme. Many 
olfactory responses are common to both sexes, such as those to many host plant volatiles, and this is reflected in the third of OR genes that we found equally expressed in both sexes. Others, particularly those involved in mating or oviposition behaviours, are likely to be sexspecific. Using RT-qPCR, we found a strong bias towards males in the number of OR genes with sex-specific expression, 15 showing significantly more expression in males and only 2 being more expressed in females. Figure 4 summarises sex differences in olfactory gene expression. The majority of genes fall into the area where sex differences in expression are less than ten-fold but 4 ORs were at least ten-fold as much expressed in males. This imbalance may be associated with male responsiveness to female sex pheromone, yet the number of ORs that show male-biased expression is well in excess of the 5 putative pheromone receptors we identified.

Our EAG studies support the conclusion that sexbiased expression of ORs is related to function and, at least in part, to the male response to sex pheromone. The EAG measures the sum of neuronal activity in the antennae reflecting the integrated response of olfactory receptors to a volatile, other related genes might also be involved in that responses. Of the 58 volatiles tested by EAG, 19 evoked a significantly sex-biased response and in each case greater electrical activity was recorded in male antenna. Of these, the strongest EAG responses were to the two behaviorally-active sex pheromone components, Z9E11-14:OAc, Z9E12-14:OAc. The two highly-expressed receptors found here, OR14 and OR23, showed strongly male-biased (Figure $3 \mathrm{~B}$ ) and it is possible that they are the receptors for Z9E11-14:OAc and Z9E12-14:OAc. Further studies are to be performed to confirm these two receptors are the receptors respond to pheromone components. Many ORs that were not candidate pheromone receptors also showed malebiased expression (Figure 2). An increase in the expression of genes that don't have a sex-specific function might in part be a by-product of the elaboration of the male antenna that enables it to carry abundant pheromone-sensitive sensilla. The sex-biased expression of CSPs (10 male-biased, 8 female-biased) indicate that CSPs play differential roles in the male and female moths. There was no sex bias in the number of OBP genes that showed sex-specific expression, probably reflecting their less specific role as binding proteins. However, the most highly expressed OBP was a pheromone binding protein, $P B P 1$, which was ten times more expressed in males, suggesting that it is involved in detection of the sex pheromone. Moreover, our data obtained from the field trials showed that some of S. litura pheromone isomers play a sygnergistic or antagonistic role when mixed into the sex pheromone blend (Du et al., unpublished result). We infers that the ORs or
OBPs could be related to recognition of those pheromone isomers and the interspecific communications.

\section{Conclusions}

In summary, we have identified the 26 olfactory receptor genes, 21 odorant-binding protein genes, 18 chemosensory protein genes and 9 ionotropic receptor genes that are key to understanding the molecular basis of olfactory responses to sex pheromones and plant volatiles in $S$. litura. Transcriptome and expression profiling analyses revealed variation in gene expression, often sex-biased, that was reflected in the strength of antennal responses and may lead to the functional identification of genes. Our results pave the way for future elucidation of the molecular basis of olfactory and mating behaviors of this moth, and the development of new biorational pheromone technologies that target particular genes, proteins and behaviors for pest monitoring and control.

\section{Methods}

Insects

Spodoptera litura (Lepidotera, Noctuidae) pupae were purchased from the Institute of Zoology, Chinese Academy of Science, and lab reared. For details see Additional file 1: Materials and Methods.

\section{EAG recording}

Recordings of whole-antenna electrical activity in response to volatile stimuli were made according to standard techniques $[64,65]$. Antennae were challenged with 58 volatile chemicals presented singly and selected from flowers, host or non-host plants, and the sex pheromone components of S. litura and their isomers, some of which are sex pheromones of other moths (Additional file 1: Table S1). Each chemical was dissolved in paraffin oil and tested at two concentrations, $10^{-4} \mathrm{v} / \mathrm{v}$ and $10^{-2}$ $\mathrm{v} / \mathrm{v}$. A $10-\mu \mathrm{l}$ aliquot of paraffin oil on the filter paper was used as the control. The responses of antennae from ten male and ten female moths were tested for each treatment. For further details see Additional file 1: Materials and Methods.

\section{Extraction of total RNA from tissues}

To obtain complete gene expression information in the transcriptome analysis, RNA was extracted separately from different developmental stages and sexes and then pooled. Separate RNA extracts were made of the antennae of each sex for expression profiling analysis and for RT-qPCR. Total RNA was extracted using RNAiso Plus (Takara, China). For further details see Additional file 1: Materials and Methods. 


\section{Transcriptome de novo analysis}

The cDNA libraries for transcriptome analysis were prepared using TruSeq SBS Kit v3-HS (Illumina, America) following manufacturer's recommendations. The libraries were sequenced using Illumina HiSeq ${ }^{\mathrm{Tm}} 2000$ (Illumina, America) with $90 \mathrm{bp}$ read length of reads-paired end. Dirty reads containing adapters and unknown or low quality bases were discarded from raw reads to obtain clean reads for analysis. Transcriptome de novo assembly was carried out with the short reads assembling program, Trinity [66]. Blastx alignment (E value $<0.00001$ ) between unigenes and protein databases (NCBI non-redundant protein database, Swiss-Prot, KEGG and COG) was successively performed. When a unigene could not be aligned to any of the databases, ESTScan software was used to decide its sequence direction and the predicted coding region [67]. Gene ontology (GO) annotations of the unigenes were determined using Blast2go (https:// www.blast2go.com/) [68]. WEGO software was used for GO functional classification for all unigenes and to understand the distribution of gene function at the macro level [69]. The raw sequence of the transcriptome has been deposited in the National Center for Biotechnology Information (NCBI) (accession number: PRJNA273435; http:// www.ncbi.nlm.nih.gov/bioproject/273435). For further details see Additional file 1: Materials and Methods.

\section{Olfactory gene analysis}

The candidate olfactory gene was obtained from GO annotation. In addition, a 50\% ORF length cutoff was used for considering a putative gene to prevent a gene from being counted twice. Amino acid sequence alignment were performed using clustalx [70]. For the phylogenetic analysis, amino acid sequences of ORs, CSPs and OBPs of D. melanogaster [22,38], Apis mellifera [71], Acyrthosiphon pisum [72], Bombyx mor [8,11], Manduca sexta [73], Spodoptera littoralis [18,51] and Heliothis virescens [14,32], and IRs of D. melanogaster [36], Bombyx mor [25], Manduca sexta [73], Spodoptera littoralis [18,51] and Helicoverpa armigera [37] were used. Phylogenetic analyses were conducted with maximum likelihood method of MEGA 6.0 based on Jones-Taylor-Thornton (JTT) substitution model, partial deletion gaps with 95\% site coverage cutoff and Nearest Neighbour Interchanges (NNI) heuristic search [74]. Node support of phylogenetic tree was assessed using the bootstrap method with 100 bootstrap replicates.

\section{Profiling analysis of antennal gene expression using single-end RNA-Seq library}

Clean reads were mapped to de novo library sequences using SOAP2 [75]. Sequence saturation analysis was used to measure the sequencing data. The distribution of reads locating on reference genes was used to evaluate the randomness of fragmentations [76]. The gene expression level was calculated using the RPKM method [77] to take account of differing gene lengths. The raw sequence has been deposited in NCBI as above. For further details see Additional file 1: Materials and Methods.

\section{RT-qPCR analysis of olfactory gene expression in antennae}

RT-qPCR was performed on total RNA of male and of female antennae to validate between-sex comparisons of gene expression made using single-end RNA-Seq data and extend them to all candidate olfactory genes, including those with lower expression levels. The PCR primers used are listed in Additional file 1: Table S3. Six or more replicates were made. The data were analyzed using SPSS 17.0. For further details see Additional file 1: Materials and Methods.

\section{Statistical analysis}

Data analysis was conducted using SAS 9.2. Significance of the difference between means was determined by Student's $t$-test.

\section{Additional file}

Additional file 1: Supporting information.

\section{Abbreviations}

E9-14:OAc: 9E-tetradecenyl acetate; Z9-14:OAc: 9Z-tetradecenyl acetate; Z9E11-14:OAc: (9Z,11E)-tetradecadienyl acetate; Z9E12-14:OAc: (9Z,12E)tetradecadienyl acetate; E11-14:OAc: 11E-tetradecenyl acetate; Z11-14: OAc: 11Z-tetradecenyl acetate; ABPs: Antennal-binding proteins; CSPs: Chemosensory proteins; EAG: Electroantennogram; FDR: False discovery rates; GO: Gene ontology; GOBPs: General odorant-binding proteins; IRs: Ionotropic receptors; ORs: Olfactory receptors; ORCO: Olfactory receptor coreceptors; OBPs: Odorant-binding proteins; PBPs: Pheromone-binding proteins; RT-qPCR: Reverse transcription-quantitative real-time PCR; RPKM: Reads Per Kb per Million reads.

\section{Competing interest}

The authors declare that they have no competing interests.

\section{Authors' contributions}

BF performed the statistical analysis, and participated in the design of the study, the transcriptome analysis and wrote part of manuscript. XL participated in the design of the study and revised the early manuscript. KZ performed the RT-qPCR and participated in the transcriptome analysis. KQ carried out the electroantennogram (EAG) recording. YC revised the draft of manuscript. YD proposed the project, designed the whole study, wrote and finalized the manuscript. All authors read and approved the final manuscript.

\section{Acknowledgements}

We are grateful to Prof. Shizhong Xu (University of California Riverside) for his help in the statistical analysis. This work was supported financially by the Special Fund for Agro-scientific Research in the Public Interest in China (Grant No. 201203036) and National 973 Project (Project no. 2012CB11410005) to YD.

\section{Author details}

${ }^{1}$ Institute of Health and Environmental Ecology, Wenzhou Medical University, University Town, Wenzhou 325035, China. ${ }^{2}$ College of Life Sciences, China Jiliang University, Hangzhou, Zhejiang 310018, China. ${ }^{3}$ Division of Neurobiology, Barrow Neurological Institute, St. Joseph's Hospital and Medical Center, Phoenix, AZ 85013, USA. 
Received: 27 November 2014 Accepted: 21 February 2015 Published online: 07 April 2015

\section{References}

1. Martin JP, Beyerlein A, Dacks AM, Reisenman CE, Riffell JA, Lei H. The neurobiology of insect olfaction: sensory processing in a comparative context. Prog Neurobiol. 2011;95(3):427-47.

2. Lei $H$, Vickers $N$. Central processing of natural odor mixtures in insects. J Chem Ecol. 2008;34(7):915-27.

3. Jaquiéry J, Stoeckel S, Nouhaud P, Mieuzet L, Mahéo F, Legeai F, et al. Genome scans reveal candidate regions involved in the adaptation to host plant in the pea aphid complex. Mol Ecol. 2012;21(21):5251-64.

4. Carlson JR. Olfaction in Drosophila: from odor to behavior. Trends Genet. 1996;12(5):175-80.

5. Witzgall $P$, Kirsch $P$, Cork A. Sex pheromones and their impact on pest management. J Chem Ecol. 2010;36(1):80-100.

6. El-Sayed AM, Suckling DM, Byers JA, Jang EB, Wearing CH. Potential of "Iure and kill" in long-term pest management and eradication of invasive species. J Econ Entomol. 2009;102(3):815-35.

7. Cook SM, Khan ZR, Pickett JA. The use of push-pull strategies in integrated pest management. Annu Rev Entomol. 2007;52(1):375-400.

8. Tanaka K, Uda Y, Ono Y, Nakagawa T, Suwa M, Yamaoka R, et al. Highly selective tuning of a silkworm olfactory receptor to a key mulberry leaf volatile. Curr Biol. 2009;19(11):881-90.

9. Nakagawa T, Sakurai T, Nishioka T, Touhara K. Insect sex-pheromone signals mediated by specific combinations of olfactory receptors. Science. 2005;307:1638-42.

10. Sakurai T, Mitsuno H, Haupt SS, Uchino K, Yokohari F, Nishioka T, et al. A single sex pheromone receptor determines chemical response specificity of sexual behavior in the silkmoth Bombyx mori. PLoS Genet. 2011;7(6):e1002115.

11. Xia Q, Zhou Z, Lu C, Cheng D, Dai F, Li B, et al. A draft sequence for the genome of the domesticated silkworm (Bombyx mori). Science. 2004;306(5703):1937-40.

12. Van Hiel M, Van Wielendaele $P$, Temmerman L, Van Soest S, Vuerinckx K, Huybrechts $R$, et al. Identification and validation of housekeeping genes in brains of the desert locust Schistocerca gregaria under different developmental conditions. BMC Mol Biol. 2009;10(1):56.

13. Krieger J, Gondesen I, Forstner M, Gohl T, Dewer Y, Breer H. HR11 and HR13 receptor-expressing neurons are housed together in pheromone-responsive sensilla trichodea of male Heliothis virescens. Chem Senses. 2009;34(6):469-77.

14. Krieger J, Grosse-Wilde E, Gohl T, Dewer YME, Raming K, Breer H. Genes encoding candidate pheromone receptors in a moth (Heliothis virescens). P Natl Acad Sci USA. 2004;101(32):11845-50.

15. Kakumani PK, Malhotra P, Mukherjee SK, Bhatnagar RK. A draft genome assembly of the army worm, Spodoptera frugiperda. Genomics. 2014;104(2):134-43.

16. Syed Z, Kopp A, Kimbrell DA, Leal WS. Bombykol receptors in the silkworm moth and the fruit fly. P Natl Acad Sci USA. 2010;107(20):9436-9.

17. Tittiger C. Functional genomics and insect chemical ecology. J Chem Ecol. 2004;30(12):2335-58.

18. Jacquin-Joly E, Legeai F, Montagné N, Monsempes C, François M, Poulain J, et al. Candidate chemosensory genes in female antennae of the noctuid moth Spodoptera littoralis. Int J Biol Sci. 2012;8(7):1036-50

19. Hallem EA, Carlson JR. Coding of odors by a receptor repertoire. Cell. 2006;125(1):143-60

20. Mitsuno H, Sakurai T, Murai M, Yasuda T, Kugimiya S, Ozawa R, et al. Identification of receptors of main sex-pheromone components of three Lepidopteran species. Eur J Neurosci. 2008;28(5):893-902.

21. Touhara K, Vosshall L. Sensing odorants and pheromones with chemosensory receptors. Annu Rev Physiol. 2009;71:307-32.

22. Leal WS. Odorant reception in insects: roles of receptors, binding proteins, and degrading enzymes. Annu Rev Entomol. 2013;58(1):373-91.

23. Zhou JJ, Vieira FG, He XL, Smadja C, Liu R, Rozas J, et al. Genome annotation and comparative analyses of the odorant-binding proteins and chemosensory proteins in the pea aphid Acyrthosiphon pisum. Insect Mol Biol. 2010;19:113-22

24. Mosbah A, Campanacci V, Lartigue A, Tegoni M, Cambillau C, Darbon H. Solution structure of a chemosensory protein from the moth Mamestra brassicae. Biochem J. 2003;369(1):39-44.
25. Olivier V, Monsempes C, François MC, Poivet E, Jacquin-Joly E. Candidate chemosensory ionotropic receptors in a Lepidoptera. Insect Mol Biol. 2011;20(2):189-99.

26. Reed RR. Signaling pathways in odorant detection. Neuron. 1992;8(2):205-9.

27. Larsson MC, Domingos Al, Jones WD, Chiappe ME, Amrein H, Vosshall LB. Or83b encodes a broadly expressed odorant receptor essential for Drosophila olfaction. Neuron. 2004;43(5):703-14.

28. Vogt R, Riddiford L. Pheromone binding and inactivation by moth antennae. Nature. 1981;293:161-3.

29. Vogt R, Rybczynski R, Lerner M. Molecular cloning and sequencing of general odorant-binding proteins GOBP1 and GOBP2 from the tobacco hawk moth Manduca sexta: comparisons with other insect OBPs and their signal peptides. J Neurosci. 1991;11(10):2972-84.

30. Vogt RG, Prestwich GD, Lerner MR. Odorant-binding-protein subfamilies associate with distinct classes of olfactory receptor neurons in insects. J Neurobiol. 1991;22(1):74-84.

31. Krieger J, von Nickisch-Rosenegk E, Mameli M, Pelosi P, Breer H. Binding proteins from the antennae of Bombyx mori. Insect Biochem Molec. 1996;26(3):297-307.

32. Krieger J, Gänßle H, Raming K, Breer H. Odorant binding proteins of Heliothis virescens. Insect Biochem Molec. 1993;23(4):449-56.

33. Raming K, Krieger J, Breer H. Primary structure of a pheromone-binding protein from Antheraea pernyi: homologies with other ligand-carrying proteins. J Comp Physiol B. 1990;160(5):503-9.

34. Angeli S, Ceron F, Scaloni A, Monti M, Monteforti G, Minnocci A, et al. Purification, structural characterization, cloning and immunocytochemical localization of chemoreception proteins from Schistocerca gregaria. Eur J Biochem. 1999;262(3):745-54.

35. Jacquin-Joly E, Vogt RG, François M-C, Nagnan-Le Meillour P. Functional and expression pattern analysis of chemosensory proteins expressed in antennae and pheromonal gland of Mamestra brassicae. Chem Senses. 2001;26(7):833-44.

36. Benton R, Vannice KS, Gomez-Diaz C, Vosshall LB. Variant ionotropic glutamate receptors as chemosensory receptors in Drosophila. Cell. 2009;136(1):149-62.

37. Liu N-Y, Xu W, Papanicolaou A, Dong S-L, Anderson A. Identification and characterization of three chemosensory receptor families in the cotton bollworm Helicoverpa armigera. BMC Genomics. 2014;15(1):597.

38. Croset V, Rytz R, Cummins SF, Budd A, Brawand D, Kaessmann H, et al. Ancient protostome origin of chemosensory ionotropic glutamate receptors and the evolution of insect taste and olfaction. PLoS Genet. 2010;6(8):e1001064.

39. Zhou Z-S, Xu Z-F, Chen Z-P. Co-efficacy of a trap crop, Colocasia esculenta (L.) Schott and a biological agent, Spodoptera litura nuclear polyhedral virus on the tobacco caterpillar, Spodoptera litura (Fabricius) in the tobacco field. Pakistan J Zool. 2011;43(4):689-99.

40. Zhou Z. A review on control of tobacco caterpillar, Spodoptera litura. Chin Bull Entomol. 2009;46(3):354-61.

41. Sun F, Hu YY, Du JW. The sex pheromone communication system of Spodoptera litura ( Fabricius). Acta Entomol Sinica. 2002;45(3):404-7.

42. Shen YL, Gao Y, Du YJ. The synergism of plant volatile compounds and sex pheromones of the tobacco cutworm moth, Spodoptera litura (Lepidoptera: Noctu idae). Acta Entomol Sinica. 2009;52(12):1290-7.

43. Chen X, Nan WZ, Jun DY, Chuan ZQ. Expression profiling of olfactory receptor gene II in the tobacco cutworm, Spodoptera litura ( Lepidoptera: Noctuidae). Acta Entomol Sinica. 2011;54(8):881-6.

44. Wu ZN, Du YJ, ZhuGe QC. Expression and localization analysis of general odorant binding protein 1 ( GOBP1) gene in Spodoptera litura (Lepidoptera: Noctuidae). Acta Entomol Sinica. 2009;52(6):610-6.

45. Wu Z-N, Chen X, Du Y-J, Zhou J-J, ZhuGe Q-C. Molecular identification and characterization of the Orco orthologue of Spodoptera litura. Insect Sci. 2013;20(2):175-82.

46. Xiu WM, Zhou YZ, Dong SL. Molecular characterization and expression pattern of two pheromone-binding proteins from Spodoptera litura (Fabricius). J Chem Ecol. 2008;34(4):487-98.

47. Galeano E, Vasconcelos T, Ramiro D, De Martin V, Carrer H. Identification and validation of quantitative real-time reverse transcription PCR reference genes for gene expression analysis in teak (Tectona grandis L.f.). BMC Research Notes. 2014;7(1):464.

48. Lu Y, Yuan M, Gao X, Kang T, Zhan S, Wan H, et al. Identification and Validation of Reference Genes for Gene Expression Analysis Using 
Quantitative PCR in Spodoptera litura (Lepidoptera: Noctuidae). PLoS One. 2013;8(7):e68059.

49. Bustin SA, Benes V, Garson JA, Hellemans J, Huggett J, Kubista M, et al. The MIQE Guidelines: Minimum Information for Publication of Quantitative Real-Time PCR Experiments. Clin Chem. 2009;55(4):611-22.

50. Legeai F, Malpel S, Montagne N, Monsempes C, Cousserans F, Merlin C, et al. An Expressed Sequence Tag collection from the male antennae of the Noctuid moth Spodoptera littoralis: a resource for olfactory and pheromone detection research. BMC Genomics. 2011;12(1):86.

51. Poivet E, Gallot A, Montagné N, Glaser N, Legeai F, Jacquin-Joly E. A comparison of the olfactory gene repertoires of adults and larvae in the noctuid moth Spodoptera littoralis. PLoS One. 2013;8(4):e60263.

52. Grosse-Wilde E, Gohl T, Bouche E, Breer H, Krieger J. Candidate pheromone receptors provide the basis for the response of distinct antennal neurons to pheromonal compounds. Eur J Neurosci. 2007;25(8):2364-73.

53. McKenzie S, Oxley P, Kronauer D. Comparative genomics and transcriptomics in ants provide new insights into the evolution and function of odorant binding and chemosensory proteins. BMC Genomics. 2014;15(1):718

54. Jones WD, Nguyen T-AT, Kloss B, Lee KJ, Vosshall LB. Functional conservation of an insect odorant receptor gene across 250 million years of evolution. Curr Biol. 2005;15(4):R119-21.

55. Benton R, Sachse S, Michnick SW, Vosshall LB. Atypical membrane topology and heteromeric function of Drosophila odorant receptors in vivo. PLoS Biol. 2006:4(2):e20.

56. Stengl M, Funk $N$. The role of the coreceptor Orco in insect olfactory transduction. J Comp Physiol A. 2013;199(11):897-909.

57. Pelosi P, Maida R. Odorant-binding proteins in insects. Comp Biochem Physiol B Biochem Mol Biol. 1995;111:503-14.

58. Vasquez GM, Fischer P, Grozinger CM, Gould F. Differential expression of odorant receptor genes involved in the sexual isolation of two Heliothis moths. Insect Mol Biol. 2011;20(1):115-24.

59. Vásquez GM, Syed Z, Estes PA, Leal WS, Gould F. Specificity of the receptor for the major sex pheromone component in Heliothis virescens. J Insect Sci. 2013;13(1):1-12.

60. Wang G, Vasquez GM, Schal C, Zwiebel LJ, Gould F. Functional characterization of pheromone receptors in the tobacco budworm Heliothis virescens. Insect Mol Biol. 2011;20(1):125-33.

61. Vogel H, Heidel A, Heckel D, Groot A. Transcriptome analysis of the sex pheromone gland of the noctuid moth Heliothis virescens. BMC Genomics. 2010;11(1):29.

62. Liu C, Liu Y, Walker WB, Dong S, Wang G. Identification and functional characterization of sex pheromone receptors in beet armyworm Spodoptera exigua (Hübner). Insect Biochem Molec. 2013;43(8):747-54.

63. Leary GP, Allen JE, Bunger PL, Luginbill JB, Linn CE, Macallister IE, et al. Single mutation to a sex pheromone receptor provides adaptive specificity between closely related moth species. P Natl Acad Sci USA. 2012;109(35):14081-6.

64. Hayase S, Renou M, Itoh T. Possible origin of modified EAG activity by point-fluorination of insect pheromones. Future Med Chem. 2009;1(5):835-45.

65. Quero C, Vidal B, Guerrero A. EAG responses increase of Spodoptera littoralis antennae after a single pheromone pulse. Nat Prod Commun 2014;9(8):1099-101.

66. Grabherr MG, Haas BJ, Yassour M, Levin JZ, Thompson DA, Amit I, et al. Full-length transcriptome assembly from RNA-Seq data without a reference genome. Nat Biotech. 2011;29(7):644-52.

67. Iseli C, Jongeneel CV, Bucher P. ESTScan: a program for detecting, evaluating, and reconstructing potential coding regions in EST sequences. In: Proc Int Conf Intell Syst Mol Biol: August 6-10, 1999; Heidelberg, Germany: AAAI Press; 1999:138-148

68. Conesa A, Gotz S, Garcia-Gomez JM, Terol J, Talon M, Robles M. Blast2GO: a universal tool for annotation, visualization and analysis in functional genomics research. Bioinformatics. 2005;21(18):3674-6.

69. Ye J, Fang L, Zheng H, Zhang Y, Chen J, Zhang Z, et al. WEGO: a web tool for plotting GO annotations. Nucleic Acids Res. 2006;34 suppl 2:W293-7.

70. Thompson JD, Gibson TJ, Plewniak F, Jeanmougin F, Higgins DG. The CLUSTAL_X windows interface: flexible strategies for multiple sequence alignment aided by quality analysis tools. Nucleic Acids Res. 1997:25(24):4876-82
71. Consortium THGS. Insights into social insects from the genome of the honeybee Apis mellifera. Nature. 2006;443(7114):931-49.

72. The International Aphid Genomics C. Genome sequence of the pea aphid Acyrthosiphon pisum. PLoS Biol. 2010;8(2):e1000313.

73. Grosse-Wilde E, Kuebler LS, Bucks S, Vogel H, Wicher D, Hansson BS. Antennal transcriptome of Manduca sexta. P Natl Acad Sci USA. 2011;108(18):7449-54.

74. Tamura K, Stecher G, Peterson D, Filipski A, Kumar S. MEGA6: molecular evolutionary genetics analysis version 6.0. Mol Biol Evol. 2013;30(12):2725-9.

75. Li R, Yu C, Li Y, Lam T-W, Yiu S-M, Kristiansen K, et al. SOAP2: an improved ultrafast tool for short read alignment. Bioinformatics. 2009;25(15):1966-7.

76. Wang Z, Gerstein M, Snyder M. RNA-Seq: a revolutionary tool for transcriptomics. Nat Rev Genet. 2009:10(1):57-63.

77. Mortazavi A, Williams BA, McCue K, Schaeffer L, Wold B. Mapping and quantifying mammalian transcriptomes by RNA-Seq. Nat Methods. 2008:5(7):621-8.

\section{Submit your next manuscript to BioMed Central and take full advantage of:}

- Convenient online submission

- Thorough peer review

- No space constraints or color figure charges

- Immediate publication on acceptance

- Inclusion in PubMed, CAS, Scopus and Google Scholar

- Research which is freely available for redistribution 